\title{
Editor's Note: Consensus statements as a variant of classical statistical methods
}

Andrew S. Wechsler, MD, Editor

See related article on page 283 .
J Thorac Cardiovasc Surg 2006;132:223

$0022-5223 / \$ 32.00$

Copyright $\odot 2006$ by The American Association for Thoracic Surgery

doi:10.1016/j.jtcvs.2006.03.026
7 he Journal has used editorials as teaching exercises as new methods of statistical analysis have been introduced as tools for understanding and testing data acquired during investigations. We have discussed propensity analysis, the use of actual and actuarial methods, and meta-analyses. There are, however, instances in which traditional statistical analyses provide steps along the path to understanding pieces of a larger puzzle but fall short of helping us achieve a broader vision of that which we are studying. Oftentimes, traditional statistical analyses yield conflicting conclusions to the same issues under study. This is not the consequence of flawed statistical method, but rather the consequence of subtle differences in study designs. Subjective terms such as context and nuance facilitate the transition from results of individual studies to broad applicability. In other words, an interpretative component allows us to apply many individual, but different, studies to everyday practice.

It is in this spirit that the work of Likosky and his colleagues is published in this issue of the Journal on page 283. The method used is "consensus," which is a distinctly non-statistical term but represents the methodology upon which most position statements are assembled. Consensus uses grades of evidence but only after those providing the consensus opinions have been free to discard or incorporate specific scientific contributions. So, whereas many studies are faulted for introducing bias at the inception of the study, in consensus opinion documents one accepts that bias exists and allows members of the consensus panel to weigh various pieces of evidence as having greater or lesser value ... as defined by the panel members.

Thus, the manuscript by Likosky and colleagues published in this issue of the Journal almost certainly is influenced by bias, and the designation of evidence grade is a combination of literature analysis and the bias of the experts involved in the study. This method incorporates a technique that has been occasionally described as "eminence" rather than "evidence," and the final product is a combination of the two. It is in this spirit that this manuscript is published. It is definitely not an attempt to suggest guidelines but rather an opportunity to demonstrate another mechanism of analysis. Remember that "class of evidence" is based on a subjective compilation of objective data. The authors may disagree with my interpretation of their work, which is offered only as a caveat to readers. Ultimately, a consensus study is dependent upon the members of the consensus panel and is, therefore, a combination of objective and subjective data analysis. 